\title{
The evaporation of the charged and uncharged water drops suspended in a wind tunnel
}

\author{
Rohini V Bhalwankar, A B Sathe and A K Kamra* \\ Indian Institute of Tropical Meteorology, Pune, India. \\ *e-mail: kamra@tropmet.res.in
}

\begin{abstract}
A laboratory experiment has been performed to study the effect of ventilation on the rate of evaporation of the millimeter sized charged and uncharged water drops suspended in a vertical wind tunnel. The linear relationship, $f_{v}=0.907+0.282 X$, observed between the mean ventilation coefficient, $f_{v}$, and a non-dimensional parameter $X,\left(X=N_{\mathrm{Sc}, v}^{1 / 3} N_{\mathrm{Re}}^{1 / 2}\right.$ where $N_{\mathrm{Sc}, v}$ is Schmidt number and $N_{\operatorname{Re}}$ is Reynold's number) is in agreement with the results of earlier investigations for uncharged water drops. However, in case of charged drops carrying $10^{-10} \mathrm{C}$ of charge, this relationship gets modified to $f_{v}=0.4877+0.149 X$. Thus, the rate of evaporation of charged drops is slower than that of uncharged drops of the same size. Oscillations of the drop and the change in airflow around drops are suggested to contribute to lowering of the ventilation coefficients for charged drops. Applicability of the results to a small fraction of highly charged raindrops falling through the sub-cloud layer below thunderstorm is discussed. The relaxation time required for a ventilated drop to reach its equilibrium temperature increases with the drop size and is higher for the charged than for the uncharged drops. It is concluded that in a given distance, charged drops will evaporate less than that of uncharged drops.
\end{abstract}

\section{Introduction}

Knowledge of the growth and evaporation of water drops within the cloud and during their fall in the sub-cloud layer is important in understanding the mechanisms of the precipitation formation. The evaporation of a water drop in an airstream is determined by the rate of transfer of heat and water vapour between the drop surface and the environmental air. The mass transfer from the surface of a drop falling at finite air velocities has been treated theoretically and experimentally by several investigators. To describe the effect of forced convection on the heat and mass transport from a spherical body, Froessling (1938) used boundary layer theory and shows the dependence of evaporation rate upon the details of airflow around the drop. Kinzer and Gunn (1951) developed a theory involving a transient transfer of packets of fresh environmental air that make molecular or thermal contact with the drop, and formulated the fundamental relation to calculate the rate of evaporation for drops falling freely through a known environment. A numerical approach to study the mass transfer of a drop falling at its terminal velocity in a sub-saturated air was presented by Woo and Hamielec (1971). They computed the effect of forced convection on the transfer of water vapour in the form of local and overall Sherwood number for spherical drops with Reynold's number in the range of $0.05-300$ and for Schmidt number equal to 0.71 . The results show that the effect of ventilation on spherical drops varies strongly with the angle from the forward stagnation point and it is smallest near the curvature of the drop and greatest on the upstream side of the drop. Watts (1971), Watts and Farhi (1975) predicted the evaporation rates, equilibrium temperature and relaxation time for stationary and falling drops.

Keywords. Drop evaporation; ventilation coefficient; evaporation-effect of electrical forces.

Proc. Indian Acad. Sci. (Earth Planet. Sci.), 113, No. 2, June 2004, pp. 129-138 
To verify these theoretical results, a large number of experimental studies have been carried out. In the experiments of Frosseling (1938), Ranz and Marshall (1952a, b) and Lee and Ryley (1968) the water drops of different sizes were suspended on a rigid support which disturbs the airflow around the drop, altering their shape and the terminal velocity. Measurements were carried out by Kinzer and Gunn (1951) in a free fall system to study the effect of ventilation coefficient on freely falling water drops of different diameters. In the analysis of the data obtained in all these experiments, the drop surface temperature was not estimated but assumed to be the temperature of adiabatic saturation. Their analysis also suffered from inaccuracies in determining the values of water vapour diffusivity and terminal velocities of the water drops. The wind tunnel measurements of the evaporation of cloud drops and small raindrops of radius 20-600 $\mu \mathrm{m}$ have been carried out by Beard and Pruppacher (1971). These results are found to be in very good agreement with the numerical results of Woo and Hamielec (1971). Pruppacher and Rasmussen (1979) extended their previous wind tunnel investigations for small drops to the drops of up to $5.0 \mathrm{~mm}$ in diameter. In all these theoretical and experimental studies, water drops have been assumed as electrically neutral. Chuang and Beard (1990) have however, numerically included the effect of electrical forces acting on raindrops falling in the vertical fields and found that commonly observed electric fields in thunderstorms have only a modest effect on drop shape but somewhat higher fields for highly charged raindrops can lead to drop's instability. Kamra and Ahire (1989) observed considerable shape changes when charged drops suspended in a vertical wind tunnel are subjected to an electric field. In strongly electrified regions of thunderstorms, the magnitude of charge on a small fraction of raindrops may approach Rayleigh's limit (Gaskell et al 1978; Marshall and Winn 1982). Since the electrical forces acting on such drops may significantly influence their growth and evaporation, it is desirable to include their effect in studying their rate of evaporation.

Here, we extend Pruppacher and Rasmussen's method for uncharged drops to study charged drop's evaporation and report the results of a laboratory simulation experiment to study the effect of ventilation on the rate of evaporation and thermal relaxation time of both charged and uncharged water drops suspended in a vertical wind tunnel. Our results for uncharged drops are compared with the results of previous experiments. Applicability of these results to a small fraction of highly charged raindrops falling in the sub-cloud layer below thunderclouds is discussed.

\section{Experimental set-up}

Measurements have been carried out in a small, low-turbulence vertical wind tunnel to calculate the ventilation coefficient of the millimeter sized water drops suspended in the air stream of the tunnel. Kamra et al $(1986,1991,1993)$ describe the details of the wind tunnel used in this experiment. It consists of a blower which sucks the air, a divergent section, a reservoir, a section having a honeycomb and three wire-mesh screens, a contraction section having a cross-wired screen at its downstream-end and a test-section. Honeycomb and screens minimise the turbulence in the flow. To maintain the desired airflow pattern and velocity well above the crossed-wired screen and to exert a back pressure on the airstream, a back-pressure plate is placed about $22 \mathrm{~cm}$ above the test-section. A water drop of millimetre size could be suspended above the test-section for many minutes. The measurements of turbulence level in the airstream in test-section are done with a hot-wire anemometer (Kamra et al 1991).

Water drops of required size formed from the triply distilled water are introduced with a calibrated pippet, in the test-section of tunnel where the turbulence level in the air stream is less than $0.8 \%$ (Kamra et al 1991). Since the size of a water drop continuously changes due to its evaporation while being suspended, the flow of the air stream in the wind tunnel is continuously controlled with a variac so the drop remains suspended above the test-section of the tunnel at its terminal velocity. The drops are photographed at 1-minute interval with a Nikon- $35 \mathrm{~mm}$ still camera using a closeup lens No. 2 and ASA-400 black and white film. Bright-field illumination with a $1 \mathrm{~kW}$ projection lamp is used to take photographs of the water drops. The drop volume and therefrom the drop's equivalent radius $a_{0}$ during its evaporation is determined from these photographs. In our experiment, the equivalent radii of the drops ranged between 0.95 and $2.05 \mathrm{~mm}$. The wind tunnel is located inside an air-conditioned room and the temperature and relative humidity of the airstream is controlled between 28 and $31^{\circ} \mathrm{C}$, and 30 and $40 \%$, respectively. The temperature is measured with a mercury thermometer to an accuracy of $0.1^{\circ} \mathrm{C}$ and the relative humidity with a dew-point hygrometer. The atmospheric pressure of the air in the laboratory is about $950 \mathrm{mb}$.

In order to electrically charge the water drops one end of a wire connected to a power supply is dipped in water in the pipette. The voltage to which the wire should be raised to get a positive charge of $10^{-10} \mathrm{C}$ is calibrated for different drop sizes. The calibration is done by collecting the water drops in a copper cylinder shielded with 
another grounded cylinder outside the test-section of the wind tunnel. The copper cylinder is placed on a Teflon sheet and connected to a Keithley electrometer. The values of potential applied to get the required charge on drops of different sizes are noted and the same potentials are applied when drops are introduced in the wind tunnel. To get uncharged drops, the wire inserted in the pipette is grounded (Kamra et al 1991).

\section{Data analysis}

The rate of change of mass of a freely falling and evaporating drop is usually expressed in terms of ventilation coefficient. Our data of mass loss with time obtained from photographic measurements for charged and uncharged drops is analysed following the procedure described by Pruppacher and Rasmussen (1979) and the ventilation coefficients for drops of different sizes are calculated. If $(d m / d t)$ and $(d m / d t)_{0}$ are the rates of evaporation of a water drop falling at its terminal velocity in air and at rest, respectively, then the mean ventilation coefficient is defined by

$$
\frac{d m}{d t}=\bar{f}_{v}\left(\frac{d m}{d t}\right)_{0} .
$$

Following Beard and Pruppacher (1971), equation (1) can be written as,

$$
\bar{f}_{v}=\frac{d m / d t}{\frac{4 \pi a_{0} D_{v, a} M_{w}\left(e_{\infty}-e_{a}\right)}{R T_{f}}},
$$

when $e_{f} / p \ll 1$ where $p$ is the total air pressure. In equation (2), $T_{f}=\left(T_{\infty}+T_{a}\right) / 2$ where $T_{a}$ is the temperature at the drop surface, $T_{\infty}$ the temperature of the air stream, $M_{w}$ molecular weight of the water, $R$ the universal gas constant, $e_{\infty}=$ $R H \times e_{\mathrm{sat}}\left(T_{\infty}\right)$ the water vapour pressure of the air, $D_{v, a}$ is the diffusivity of water vapour in air at the drop surface temperature $T_{a}, e_{a}=e_{\text {sat }}\left(T_{a}\right)$, $e_{f}=\left(e_{\infty}+e_{a}\right) / 2$ and $a_{0}$ is the radius of the drop.

The values of $d m / d t$ and $a_{0}$ are determined from our photographs of the drop and $e_{\infty}$ from the dew point of the air. $D_{v, a}$ is given by Hall and Pruppacher (1976) as

$$
D_{v, a}=0.211\left(\frac{T_{a}}{T_{0}}\right)^{1.94}\left(\frac{p_{0}}{p}\right)
$$

where $p_{0}=1013.25 \mathrm{mb}$ and $T_{0}=273.25^{\circ} \mathrm{K}$.

The drop surface temperature $T_{a}$ is determined from

$$
\Delta T=T_{\infty}-T_{a}=\frac{L_{e} D_{v, a} M_{w}\left(e_{\infty}-e_{a}\right) \bar{f}_{v}}{k R T_{f} \bar{f}_{h}}
$$

where $L_{e}$ and $k$ are the latent heat of evaporation and thermal conductivity of the air at the drop's surface temperature, respectively. $\bar{f}_{h}$ is a measure for the effect of ventilation on the transfer of heat to the drop. Equation (4) has been numerically solved following Beard and Pruppacher (1971). The values of thermal conductivity of moist air and the latent heat of evaporation are computed from the equations given by Pruppacher and Rasmussen (1979).

\section{Results}

The values of the mean ventilation coefficient for water drops of $a_{0}>1 \mathrm{~mm}$ and $N_{\mathrm{Sc}, v}=0.71$ are determined from equation (2). These values are plotted against a non-dimensional parameter $X=$ $N_{\mathrm{Sc}, v}^{1 / 3} N_{\mathrm{Re}}^{1 / 2}$ where $N_{\mathrm{Sc}, v}$ is Schmidt number and $N_{\mathrm{Re}}$ is Reynold's number. The parameter $X$ gives an estimate of an aerodynamic flow around the drop. From a total of 82 raw data points obtained for uncharged drops suspended in the $30-40 \%$ relative humidity range, 14 average data points are calculated and plotted in figure 1(a). The horizontal axis in the figure also shows the relation of $X$ with size of the drop. A linear relationship is obtained between the two dimensionless quantities and the line-of-best-fit can be expressed by

$$
\bar{f}_{v}=0.9073+0.2817 X .
$$

Our results for uncharged drops are in reasonably good agreement with those of Pruppacher and Rasmussen (1979). For example, a water drop of $a_{0}=2 \mathrm{~mm}$ freely falling at its terminal velocity will evaporate 15 times faster than a drop of the same size at rest according to the results of Pruppacher and Rasmussen (1979). Results of our experiment give the value of this factor as 13.1.

Figure 1(b) shows a plot of $f_{v}$ against $X$ for drops carrying a charge of $10^{-10} \mathrm{C}$. In this case a total of 51 raw data points obtained in the same range of relative humidity are used to calculate 16 average data points. The line-of-best-fit in this case is given by,

$$
\bar{f}_{v}=0.4877+0.1489 X .
$$

The line-of-best-fit in figure $1(\mathrm{~b})$ is extended only up to $a_{0} \sim 0.3 \mathrm{~mm}$ since a charge of $10^{-10} \mathrm{C}$ is larger than the Rayleigh's limit for smaller drops. The results show that the rates of evaporation of charged drops are lesser than that of uncharged drops of the same size. For example, a charged drop of $2 \mathrm{~mm}$ radius falling at its terminal velocity evaporates only 7.08 times faster instead of 13.1 times faster in case of an uncharged drop when compared to a water drop of the same size at rest. 


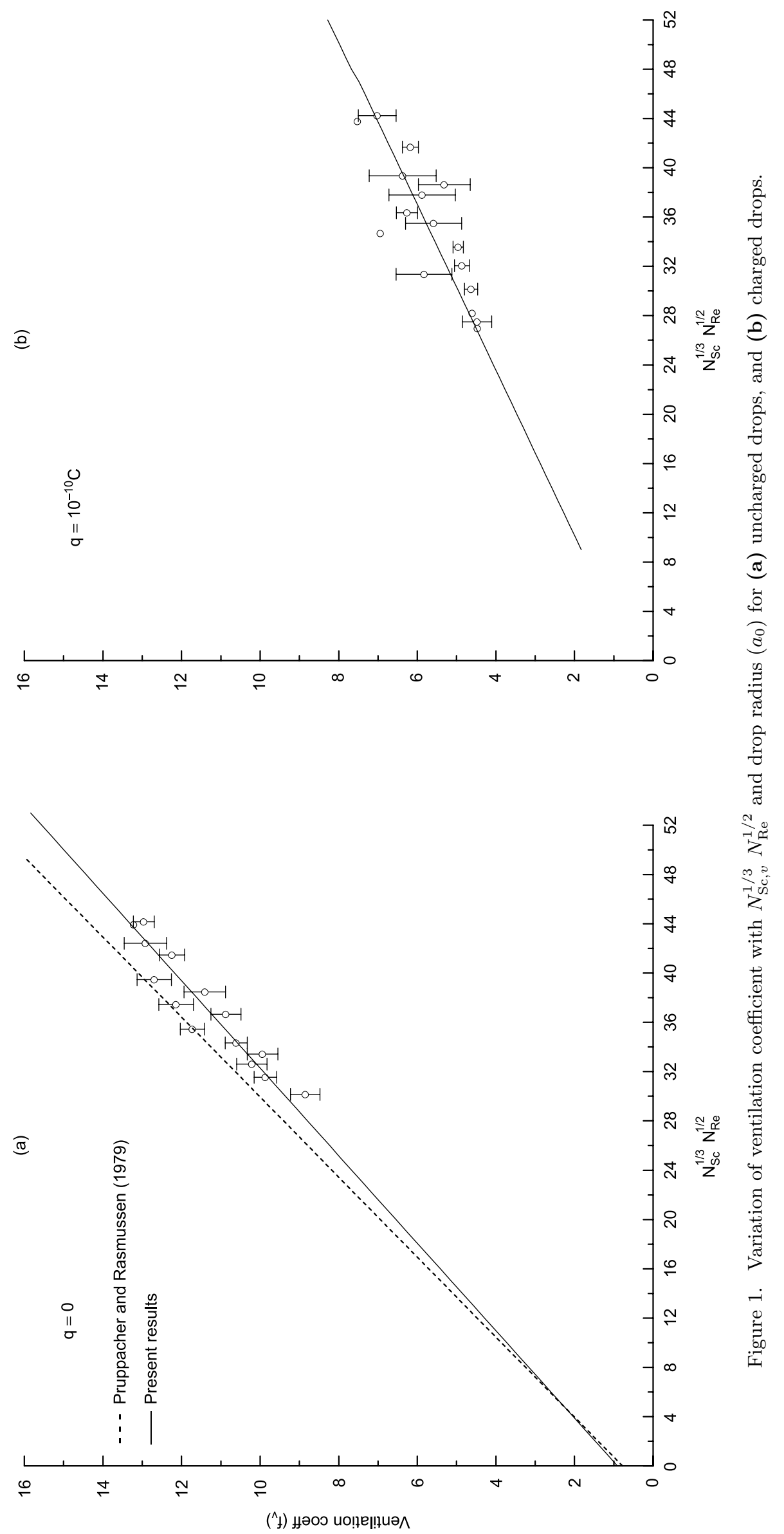


Equation (2) can be written as $Y^{\prime} Y^{\prime \prime}=-d m / d t$ where $Y^{\prime}=4 \pi a_{0} f_{v}$ and $Y^{\prime \prime}=D_{v, a}\left(e_{\infty}-e_{a}\right) /\left[R_{v} T_{f}\right]$ where $R_{v}=R / M_{w}$. $Y^{\prime}$ is computed for different drop sizes for $30 \%$ relative humidity and $T_{\infty}, 28.3^{\circ} \mathrm{C}$ for both uncharged $(q=0)$ and charged $\left(q=10^{-10} \mathrm{C}\right)$ drops. $Y^{\prime \prime}$ is sensitive to the relative humidity of the environment. Tables 1 and 2 can be used to directly calculate the change in mass

Table 1. Values of $Y^{\prime}=4 \pi a_{0} f_{v}(\mathrm{~cm})$ for charged and uncharged drops as a function of drop diameter with ambient temperature $28.3^{\circ} \mathrm{C}$.

\begin{tabular}{cccc}
\hline S. No. & $\begin{array}{c}\text { Diameter } \\
(\mathrm{mm})\end{array}$ & $\begin{array}{c}Y^{\prime}(q=0) \\
(\mathrm{cm})\end{array}$ & $\begin{array}{c}Y^{\prime}\left(q=10^{-10} \mathrm{C}\right) \\
(\mathrm{cm})\end{array}$ \\
\hline 1 & 1.0 & 3.215 & 1.704 \\
2 & 1.2 & 4.411 & 2.338 \\
3 & 1.4 & 5.755 & 3.049 \\
4 & 1.6 & 7.246 & 3.838 \\
5 & 1.8 & 8.876 & 4.701 \\
6 & 2.0 & 10.638 & 5.633 \\
7 & 2.2 & 12.523 & 6.631 \\
8 & 2.4 & 14.520 & 7.687 \\
9 & 2.6 & 16.619 & 8.798 \\
10 & 2.8 & 18.810 & 9.957 \\
11 & 3.0 & 21.085 & 11.160 \\
12 & 3.2 & 23.435 & 12.404 \\
13 & 3.4 & 25.852 & 13.682 \\
14 & 3.6 & 28.331 & 14.993 \\
15 & 3.8 & 30.864 & 16.334 \\
16 & 4.0 & 33.449 & 17.701 \\
17 & 4.2 & 36.080 & 19.092 \\
18 & 4.4 & 38.754 & 20.507 \\
19 & 4.6 & 41.470 & 21.944 \\
20 & 4.8 & 44.226 & 23.401 \\
21 & 5.0 & 47.020 & 24.879 \\
\hline & & & \\
\hline
\end{tabular}

Table 2. Values of $Y^{\prime \prime}=(-d m / d t) /\left(4 \pi a_{0} f_{v}\right)$ in $\mu g \sec ^{-1}$ $\mathrm{cm}^{-1}$ for ambient temperature $28.3^{\circ} \mathrm{C}$, pressure $950 \mathrm{mb}$, and different relative humidities.

\begin{tabular}{|c|c|c|}
\hline S. No. & $\begin{array}{c}\text { Relative humidity } \\
\%\end{array}$ & $\begin{array}{c}Y^{\prime \prime}=-(d m / d t) / 4 \pi a_{0} f_{v} \\
\mu \mathrm{g} \mathrm{sec}^{-1} \mathrm{~cm}^{-1}\end{array}$ \\
\hline 1 & 0 & 2.029 \\
\hline 2 & 10 & 1.780 \\
\hline 3 & 20 & 1.524 \\
\hline 4 & 30 & 1.287 \\
\hline 5 & 40 & 1.067 \\
\hline 6 & 50 & 0.861 \\
\hline 7 & 60 & 0.669 \\
\hline 8 & 70 & 0.488 \\
\hline 9 & 80 & 0.317 \\
\hline 10 & 90 & 0.155 \\
\hline
\end{tabular}

of drop with time for different environmental conditions. $d m / d t$ is higher for uncharged than for charged drops.

Kinzer and Gunn (1951) calculated a dimensionless quantity $F$ which they called as Ventilation Factor and is related with $f_{v}$ as, $f_{v}=1+$ $F\left(N_{\mathrm{Sc}, v} N_{\mathrm{Re}} / 4 \pi\right)^{1 / 2}$. They found that $F$ is a marked function of $N_{\mathrm{Re}}$. Dependence of $F$ on the square root of Reynold's number of the drop derived from the experimental results of Kinzer and Gunn (1951) and Beard and Pruppacher (1971) are compared with the present results for charged and uncharged drops in figure 2 . The variations in our experimental values of $F$ with $\left(N_{\mathrm{Re}}\right)^{1 / 2}$ are comparable with those of Beard and Pruppacher (1971), for uncharged drops. Beard and Pruppacher (1971) pointed out that Kinzer and Gunn (1951) set the drop temperature equal to the ambient wetbulb temperature which might be somewhat higher than the equilibrium surface temperature. Moreover, they derived values for the rate of change of mass from the variation in fall speed of an evaporating drop using double finite difference method and used inaccurate values for the drag on a drop. It may introduce error in calculating $F$ values in their computations and the error will be larger for the smaller drops. The values of $F$ are much lower for the drops carrying a charge of $10^{-10} \mathrm{C}$ than that for the uncharged drops. Such highly charged drops develop a pronounced oblate distortion caused by the charge density enhancement at their waist, (Kamra et al 1991; Chuang and Beard 1990). This change in shape of the charged drops alters the airflow around them and consequently results in a decrease in their ventilation coefficients.

The present results can be applied to compute the evaporation of raindrops falling from clouds through the subsaturated sub-cloud air of various relative humidities in a NACA standard atmosphere. Following Mason (1952) and using an iterative method, we have computed the size of drops falling below the cloud in air of various humidities and reaching the ground with a radius of $1 \mathrm{~mm}$. The changes in size of uncharged drops as a function of the distance below a cloud base are illustrated in figure 3(a). Our results are in agreement with those of Pruppacher and Rasmussen (1979) who show that the lower the relative humidity of the sub-cloud air the larger the drop has to be to reach the ground with a given size. The slight but systematic difference between the curves of Pruppacher and Rasmussen (1979) and ours may be because of the differences in the initial conditions of the two experiments. Figure 3(b) shows similar curves for the drops carrying an electrical charge of $10^{-10} \mathrm{C}$. It follows from figure $3(\mathrm{a})$ and (b) that the charged drops leaving the cloud base have to be smaller in size than the uncharged drops 


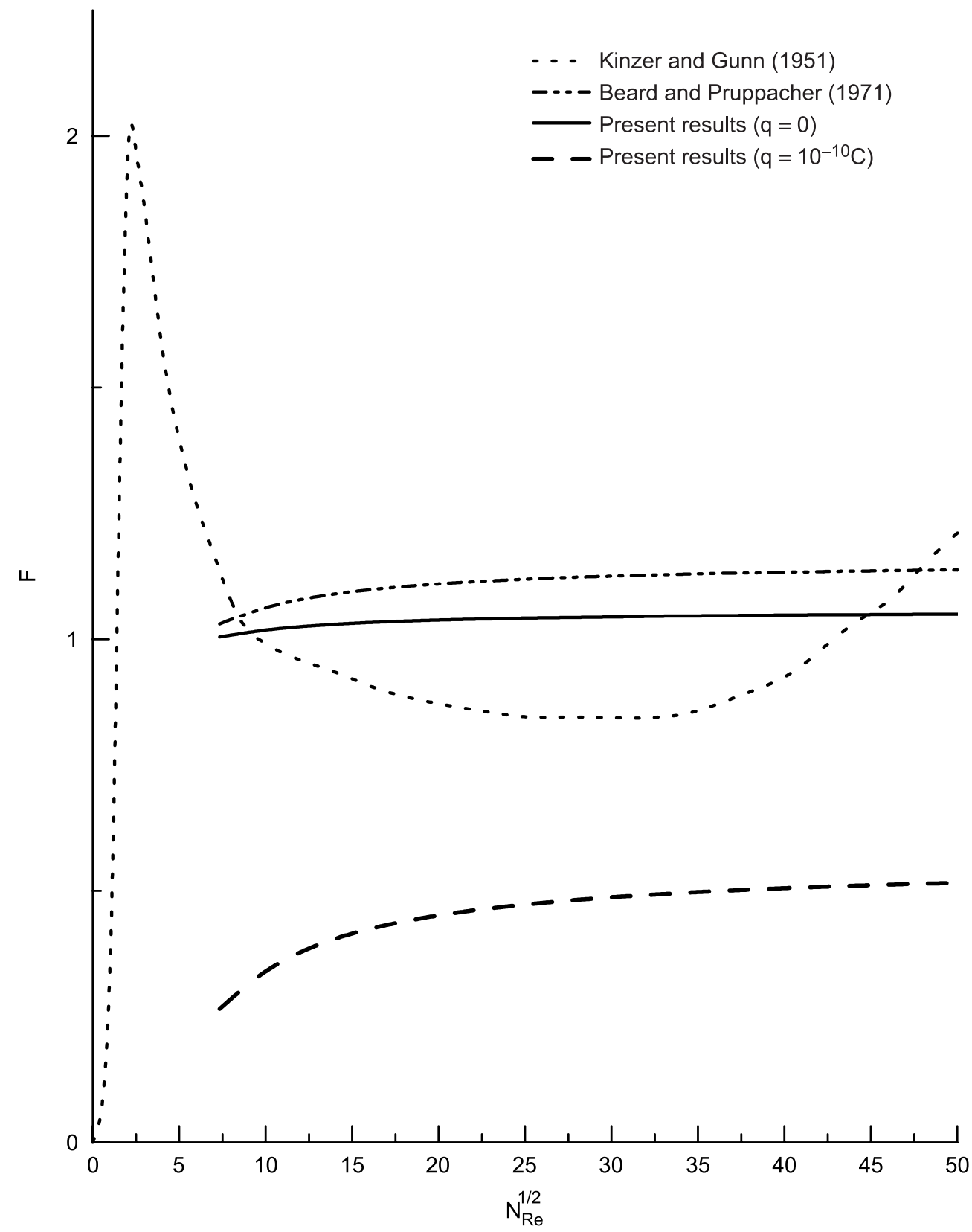

Figure 2. Dependence of ventilation factor, $F$, with the square root of Reynold's number for charged and uncharged drops.

to reach the ground with the same size. For example, the radius of a drop falling from $2 \mathrm{~km}$ height in an atmosphere of $70 \%$ relative humidity has to be of $1.07 \mathrm{~mm}$ if uncharged and $1.037 \mathrm{~mm}$ if charged, for it to reach the ground with $1 \mathrm{~mm}$ radius. So, in a given distance, charged drops will evaporate less as compared to the uncharged drops.

\section{Relaxation times of charged and uncharged drops}

On the surface of a freely falling water drop the vapour and cooled air is continuously replaced by environmental air i.e., the drop evaporates and cools. Because of this evaporative cooling the drop surface temperature is lower than the surrounding air. There is conductive heat flux from the air to the drop and mass loss from the drop due to its evaporation. This heat-mass transfer process causes a temperature difference between the drop and its environment. The time required for a ventilated drop to reach equilibrium temperature moving from one environment to another is called as relaxation or adaptation time $(\tau)$ and is given by Pruppacher and Klett (1998) as,

$$
\tau=\frac{a_{0}^{2} \rho_{w} C_{w}}{\left[3\left(k \overline{f_{h}}+L_{e} D_{v}\left(\frac{d \rho}{d T}\right)_{\text {sat }} \overline{f_{v}}\right)\right]},
$$




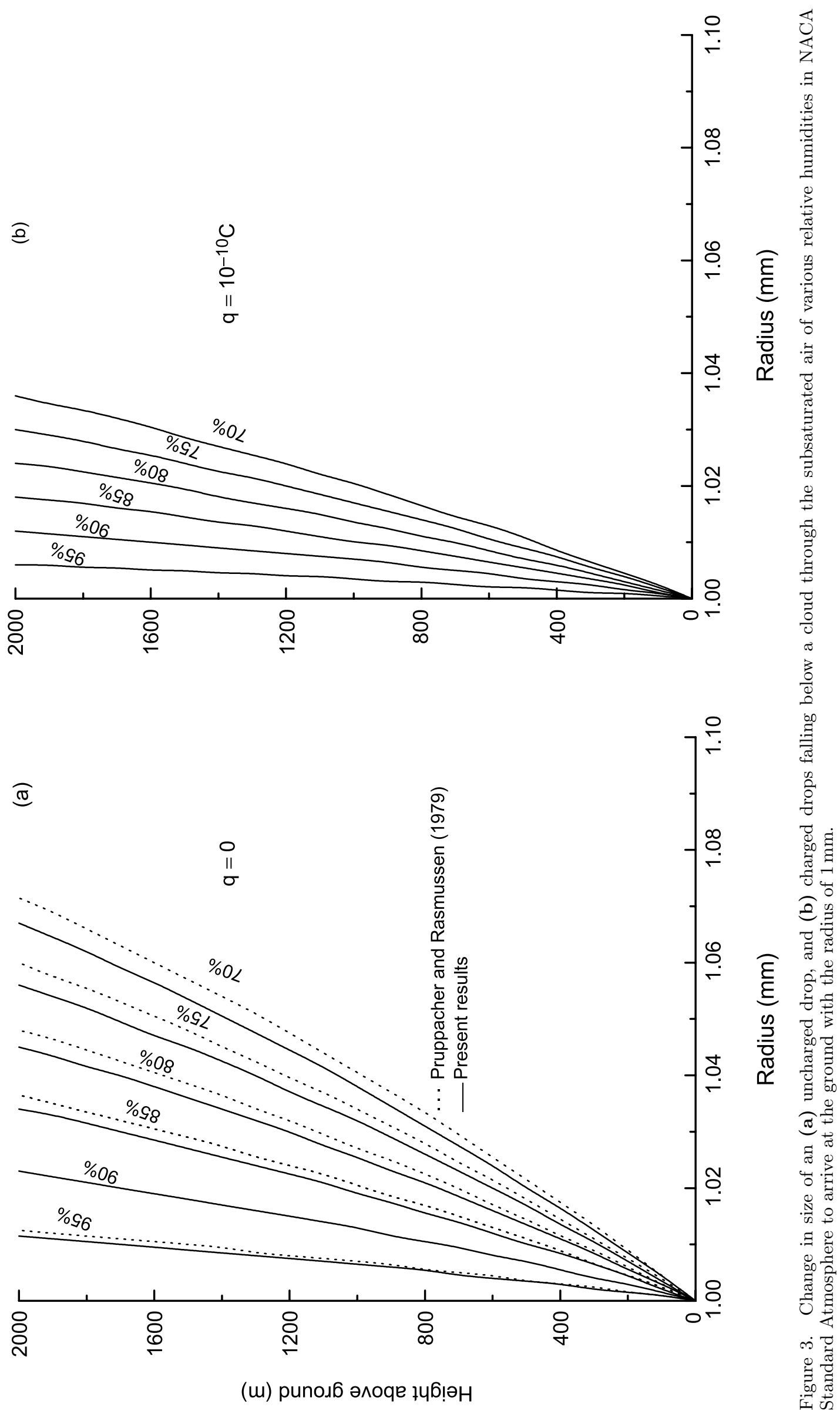




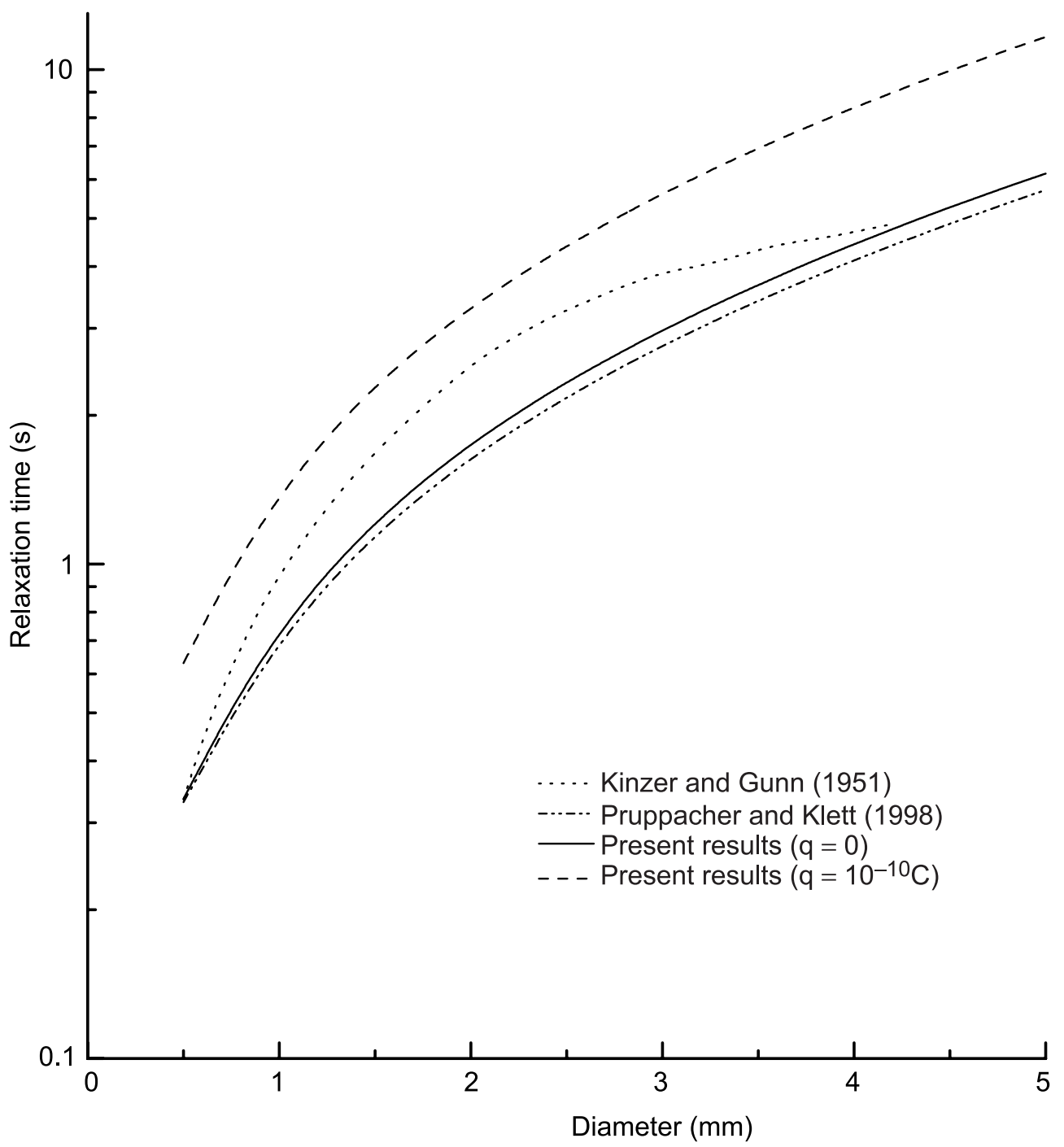

Figure 4. Relaxation time as a function of drop diameter for charged and uncharged drops.

where $\rho_{w}$ is the density of water, $C_{w}$ is the specific heat capacity of water and $\left(d \rho_{v} / d T\right)_{\text {sat }}=$ $\left[\rho_{v, \text { sat }}\left(T_{\infty}\right)-\rho_{v, \text { sat }}\left(T_{a}\right)\right] /\left(T_{\infty}-T_{a}\right)$ is the mean slope of the saturated vapour density temperature curve over the interval $T_{\infty}$ to $T_{a}$ and assuming $\bar{f}_{v}=\bar{f}_{h}$.

The values of relaxation time computed from equation (7) for the charged and uncharged drops of different sizes are plotted in figure 4 which also shows the results of Kinzer and Gunn (1951) and Pruppacher and Klett (1998), for comparison. In general, the time required to reach its final equilibrium temperature increases with the drop size. Moreover, the values of $\tau$ are higher for charged drops than for uncharged drops due to the lower evaporation rates of charged drops. For example, from our experimental values of ventilation coefficient, the estimated values of relaxation time $\tau=2.57 \mathrm{~s}$ for uncharged drop and $4.86 \mathrm{~s}$ for the charged drop $\left(10^{-10} \mathrm{C}\right)$ of radius $1.35 \mathrm{~mm}$ when these are falling in an air stream of temperature $28.3^{\circ} \mathrm{C}$ and relative humidity $30-40 \%$. The only experiment performed by Kinzer and Gunn (1951) to measure the relaxation time for a drop of radius $1.35 \mathrm{~mm}$ supported in an air column with temperature $28.3^{\circ} \mathrm{C}$ and $22 \%$ relative humidity gives $\tau=4.35 \mathrm{~s}$ which is comparable with their numerically calculated value of thermal relaxation time $\tau=3.6 \mathrm{~s}$.

\section{Discussion}

The equilibrium shape of a water drop falling at its terminal velocity in air can be determined from the balance of local interfacial forces. Progressive change in shape of the drop is observed with the increase in its size and the flow field past around it. Water drops of radius $>140 \mu \mathrm{m}$, freely falling in air, distort from their spherical shape 
and oscillate between the prolate and oblate spheroidal shapes. However, Pruppacher and Rasmussen (1979) conclude from their experimental results that the distortion, oscillation and internal circulation of large drops don't influence the effect of ventilation on the evaporation of uncharged drops since the larger effect of ventilation for a prolate spheroid than for a sphere is approximately compensated by the smaller effect of ventilation for an oblate spheroid than for a sphere. Results for uncharged drops of diameter 1.91-4.1 mm in our experiment are in conformity with their results.

Magnitude of electrical charge on some drops in the intensely electrified regions of thunderclouds may approach the Rayleigh's limit due to a variety of electrical processes (e.g., Gaskell et al 1978; Marshall and Winn 1982). In case of an oblateshaped drop, the maximum charge concentration appears around its waist where the drop curvature is maximum (Kamra et al 1991). Since the surface electric stress caused by the charge opposes the surface tension force of the drop, the effect of drop charge is to enhance its oblateness i.e., to increase the mean value of drop's major axis around which it oscillates (Kamra et al 1991). This causes an increase in the cross sectional area of the charged drop exposed to the airflow which in turn increases the drag and thus decreases the terminal velocity of the drop (Dawson and Warrender 1973; Gay et al 1974; Chuang and Beard 1990; Coquillat and Chauzy 1993).

The results of theoretical models for the values of size and charge on the drops used in our experiment do not show any significant change in the mean shape or velocity of the drop. However, in most of the theoretical models, the effects of oscillations and vibrations of drop are not included and therefore they fail to predict the extremes of drop's deformation. The theoretically predicted estimates of the mean shape are therefore an underestimate of the maximum oblateness of drops. This is especially true in case of charged drops where the electrical forces and drop distortion feedback each other to enhance the oblateness of the drop (Kamra et al 1991). Deficiency of theoretical models for not properly accounting for the effects of electrical forces is also reflected in their not being able to explain the experimental observations that,

- oscillating drops in the presence of vertical electric fields show more stretching than the model results (Richards and Dawson 1973) and,

- the curves of distortion ratio vs. electric field strength for given drop size crossover each other (Rassmussen et al 1985).

Further, in case of an oscillating charged drop, the effects of ventilation when the drop's oblate- ness is maximum or minimum around its mean value, may not exactly compensate each other as suggested by Pruppacher and Rasmussen (1979) for the uncharged drops, because the charge accumulation is maximum around the drop's waist and consequently the maximum amplitude of the oscillation of the drop around its equilibrium shape may be greater than its minimum amplitude. So, oscillations of a charged drop produce a shift in the average axis ratio toward higher values. Unfortunately, there is no high-resolution experimental data to study the effect of drop charge on its shape during its oscillations. Observations of higher probability of the spontaneous breakup of the charged than uncharged drops in wind-tunnel experiments of Kamra et al (1991), however, strongly support greater oblateness of charged drops.

Since the viscous flow past a body is critically dependent on the shape of that body, the ventilation coefficient varies with the change in its shape. Pitter et al (1974) show that at any particular Reynolds number, the sphere has the largest ventilation coefficient and it decreases with decrease in its axis ratio as the drop becomes oblate spheroid. As the Reynolds number increases, the variation in the ventilation coefficient between various shapes steadily increases.

Some factors are known to cause variation in ventilation coefficient between various shapes of the drop with $N_{\operatorname{Re}}<100$. The length of standing eddy that develops at downstream end of a drop grows in length with increasing Reynolds number and/or deformation of a drop to an oblate spheroid (Pitter et al 1973). For example, for a drop with $N_{\mathrm{Re}}=100$, the length of eddy will increase from 1.38 to 1.91 times its size when the ratio of minor to major axis of an oblate spheroid decreases from 0.5 to 0.2. If the results of Pitter et al (1973) are extrapolated for larger drops, similar increase in the eddy length will require smaller decrease in the ratio of minor to major axis of the drop. For intermediate Reynolds numbers, as the Reynolds number and/or deformation of the drop increases, the magnitude of vapour density derivative increases in the front half of the oblate drop. Increase in the derivative in the rear half of the drop is only minimal as the eddy serves as a thick boundary layer and restricts mass transport to the environmental fluid in that region (Pitter et al 1974). So, the forced convection mechanism is not able to operate efficiently over the entire surface area of the oblate drop. Therefore, the ventilation coefficient will decrease with increasing oblateness of the body. In case of drops with low to intermediate Reynolds number, Sherwood number, $N_{\mathrm{sh}}$, for oblate spheroid has been found to be less by up to $50 \%$ of that for the sphere (Brenner 1963; Woo and Hamielec 1971; Beard and Pruppacher 1971; and 
Pitter et al 1974). As pointed out by Pitter et al (1974), similar arguments can justify the extension of the results to the intermediate enhancement of thick oblate spheroids.

Most of the drops inside thunderstorms are likely to be little influenced by the above results because the value of relative humidity inside thunderstorms is mostly close to $100 \%$ and only a small fraction of drops may carry a charge in excess of $10^{-10} \mathrm{C}$. However, these results are applicable to the drops which fall close to the edges of rainshaft and undergo considerable evaporation and thus approach their charge limit. The evaporation of drops in the dry air below thunderstorms is quite important in some regions, for example, in the southwestern United States, where sometimes all the water drops falling below cloud bases evaporate before reaching the ground. This kind of rain is called 'virga'. Zrink et al (1984) suggested that the change in drop shape due to surface charge might be detected in the differential reflectivity of radar signal from electrically active thunderstorms, particularly just before the lightning. The above results also show that, some raindrops falling from an electrified thunderstorm may evaporate at a lesser rate than those falling from a weakly electrified cloud.

\section{References}

Beard K V and Pruppacher H R 1971 A wind tunnel investigation of the rate of evaporation of small water drops falling at terminal velocity in air; J. Atmos. Sci. 28 14551464

Brenner H 1963 Forced convection heat and mass transfer at small Peclet numbers from a particle of arbitrary shape; Chem. Eng. Sci. 18 109-122

Chuang C C and Beard K V 1990 A numerical model for the equilibrium shape of electrified raindrops; J. Atmos. Sci. 47 1374-1389

Coquillat S and Chauzy S 1993 Behavior of precipitating water drops under the influence of electrical and aerodynamical forces; J. Geophys. Res. 98 10,319-10,324

Dawson G A and Warrender R A 1973 The terminal velocity of raindrops under vertical electric stress; J. Geophys. Res. 78 3619-3620

Froessling N 1938 Ueber die Verdunstung fallender Tropfen. Gerlands Beitr; Z. Geophys. 52 17,216

Gay M J, Griffiths R F, Latham J and Saunders C P R 1974 The terminal velocity of charged raindrops and cloud droplets falling in strong electric fields; Q. J. R. Meteorol. Soc. $100682-687$

Gaskell W, Illingworth A J, Latham J and Moore C B 1978 Airborne studies of electric fields and the charge and size of precipitation elements in thunderstorms; $Q . J . R$. Meteorol. Soc. 104 447-460

Hall W D and Pruppacher H R 1976 The survival of ice particles falling from cirrus clouds in subsaturated air; $J$. Atmos. Sci. 32 1995-2006
Kamra A K and Ahire D V 1989 Wind tunnel studies of the shape of charged and uncharged water drops in the absence or presence of an electric field; Atmos. Res. 23 $117-134$

Kamra A K, Bhalwankar R V and Sathe A B 1991 Spontaneous breakup of charged and uncharged water drops freely suspended in a wind tunnel; J. Geophys. Res. 96 $17,159-17,168$

Kamra A K, Bhalwankar R V and Sathe A B 1993 The onset of disintegration and corona in water drops falling at terminal velocity in horizontal electric fields; J. Geophys. Res. 98 12,901-12,912

Kamra A K, Sathe A B and Ahire D V 1986 A vertical wind tunnel for water drop studies; Mausam 37 210-222

Kinzer G D and Gunn R 1951 The evaporation, temperature and thermal relaxation time of freely falling waterdrops; J. Meteorol. 8 71-83

Lee K and Ryley D J 1968 The evaporation of water drops in supersaturated steam; J. Heat Transfer 90 445451

Marshall T C and Winn W P 1982 Measurements of charged precipitation in a New Mexico Thunderstorm: Lower positive charge centres; J. Geophys. Res. 87 7141-7157

Mason B J 1952 Production of rain and drizzle by coalescence in stratiform clouds; Q. J. R. Meteor. Soc. 78 377386

Pitter R L, Pruppacher H R and Hamielec A E 1973 A numerical study of viscous flow past a thin oblate spheroid at low and intermediate Reynolds numbers; J. Atmos. Sci. 30 125-134

Pitter R L, Pruppacher H R and Hamielec A E 1974 A numerical study of the effect of forced convection on mass transport from a thin oblate spheroid of ice in air; $J$. Atmos. Sci. 31 1058-1066

Pruppacher H R and Klett J D 1998 Microphysics of Clouds and Precipitation (Dordrecht: Kluwer Academic Pub.)

Pruppacher H R and Rasmussen R 1979 A wind tunnel investigation of the rate of evaporation of large water drops falling at terminal velocity in air; J. Atmos. Sci. 36 1255-1260

Ranz W E and Marshall W R 1952a Evaporation from drops; Chem. Eng. Progr. 48 141-146

Ranz W E and Marshall W R 1952b Evaporation from drops; Chem. Eng. Progr. 48 173-180

Rasmussen R, Walcek C, Pruppacher H R, Mitra S K, Lew J, Levizzani V, Wang P K and Barth U 1985 A wind tunnel investigation of the effect of an external, vertical electric field on the shape of electrically uncharged rain drops; $J$. Atmos. Sci. 42 1647-1652

Richards C N and Dawson G A 1973 Stresses on a rain drop falling at terminal velocity in a vertical electric field; Phys. Fluids 16 796-800

Watts R C 1971 Relaxation time and steady evaporation rate of freely falling raindrops; J. Atmos. Sci. 28 219225

Watts R G and Farhi I 1975 Relaxation times for stationary evaporating liquid droplets; J. Atmos. Sci. 32 18641867

Woo S W and Hamielec A E 1971 A numerical method of determining the rate of evaporation of small drops falling at terminal velocity in air; J. Atmos. Sci. $281448-$ 1454

Zrink D S, Doviak R J and Mahapatra P R 1984 The effect of charge and electric field on the shape of rain drops; Radio Science 19 75-80 